Ciencias Agrarias/Agricultural Sciences

Cienc Tecn UTEQ (2017) 10(2) p 89-101

ISSN 1390-4051; e-ISSN 1390-4043

(c) (i) (2)

DOI: https://doi.org/10.18779/cyt.v10i2.172

\title{
Identificación de un gen codificante de polifenol oxidasa (PPO) en Theobroma cacao L. (cacao) de Ecuador
}

\section{Identification of a gene encoding polyphenol oxidase (PPO) in Theobroma cacao L. (cacao) from Ecuador}

\author{
${ }^{\triangle}$ Jaime Morante-Carriel ${ }^{1}$, Milena Acosta-Farías², Víctor Huebla-Concha ${ }^{3}$, Anna Agnieszka-Obrebska ${ }^{1}$, \\ Roque Bru-Martínez ${ }^{4}$, María Lorena Cadme-Arévalo ${ }^{1}$
}

${ }^{1}$ Universidad Técnica Estatal de Quevedo, Facultad de Ciencias Ambientales, Departamento de Biotecnología. Laboratorio de Biología Molecular, Campus Manuel Haz Álvarez. Av. Quito Km 1.5 vía Santo Domingo de los Tsáchilas. EC.120301. Quevedo, Ecuador.`jmorante@uteq.edu.ec; anna.obrebska31@gmail.com; mcadme@uteq.edu.ec

${ }^{2}$ Universidad Politécnica Salesiana (UPS), Carrera de Ingeniería en Biotecnología de los Recursos Naturales, Campus Girón, Av. Isabel La Católica N23-52 y Madrid, Quito,Ecuador.jmacostafarias@gmail.com

${ }^{3}$ Escuela Superior Politécnica de Chimborazo-ESPOCH-Extensión Morona Santiago, Macas, Ecuador. zvictorh@yahoo.es

${ }^{4}$ Universidad de Alicante, Grupo de Proteómica y Genómica Funcional de Plantas. Ctra. San Vicente s/n, 03690

San Vicente del Raspeig, Alicante, España. roque.bru@ua.es

Rec.: 05.06.2017. Acept.: 26.09.2017.

Publicado el 1 de diciembre de 2017

Resumen

$\mathrm{E}^{\prime}$ n Ecuador, las plantaciones de cacao presentan bajos promedios de producción debido a la diversidad de patógenos, especialmente a la infección por Moniliophthora roreri (monilia). Se cree que existe una relación entre el ataque del hongo y el aumento de los niveles de expresión de genes codificantes de polifenol oxidasas (PPOs) como mecanismo de defensa ante patógenos y herbívoros en diferentes plantas. Para la identificación de genes que codifican para PPOs, se seleccionaron hojas de cacao Nacional, provenientes de plantas resistentes y susceptibles a monilia, ubicadas en la Finca Experimental La Represa, propiedad de la Universidad Técnica Estatal de Quevedo. Se afinó un protocolo de extracción de ARN total de alta calidad para hojas de cacao recalcitrantes. Después de su retrotranscripción a ADNc, se realizaron ensayos de amplificación por PCR con diferentes primers, diseñados a partir de secuencias conservadas de PPOs. Los productos de amplificación permitieron la identificación de un gen de 961 $\mathrm{pb}$, similar a un gen que codifica para la PPO predictiva de Theobroma cacao depositada en NCBI (XP_017978715.1) La identificación de este gen, es fundamental para evaluar a futuro los niveles de expresión y cuantificación en diferentes estados de desarrollo del fruto. Dicha cuantificación permitirá proponer herramientas de control para monilia y construir las bases para el mejoramiento genético del cacao Nacional.

Palabras clave: polifenol oxidasas, cacao, identificación, expresión génica
Tn Ecuador, cocoa plantations have low production averages due to the diversity of pathogens, especially the infection by Moniliophthora roreri (monilia). It is believed that there is a relationship between the attack of the fungus and increased levels of expression of genes encoding polyphenol oxidases (PPOs) as a defense mechanism against pathogens and herbivores in different plants. For the identification of genes that code for PPOs, National cocoa leaves were selected, from resistant and susceptible plants to monilia, located in the Finca Experimental La Represa, property of the Universidad Técnica Estatal de Quevedo. A high-quality total RNA extraction protocol was recalculated for recalcitrant cocoa leaves. After its retrotranscription to cDNA, PCR amplification assays will be carried out with different primers, from the conserved sequences of PPOs. The amplification products allowed the identification of a 961 bp gene, similar to a gene that codes for the predictive PPO of Theobroma cacao deposited in NCBI (XP_017978715.1). The identification of this gene is essential to evaluate future levels of expression and quantification in different stages of fruit development. This reading quantification will propose control tools for monilia and build the bases for the genetic improvement of the National cacao.

Key words: polyphenol oxidases, cocoa, identification, gene expression 


\section{Introducción}

$\mathrm{L}$ a calidad de los frutos ha sido analizada por el consumidor desde tiempos inmemorables, por lo que el agricultor es presionado por los clientes para liberar al mercado productos que satisfagan sus necesidades. La calidad se valora mediante la medición de las características organolépticas, características intrínsecas, así como la apariencia del producto (Morante et al., 2014). Las PPOs (polifenol oxidasas) son importantes en la industria agro-alimentaria e influyen en la calidad de un producto, la cual disminuye considerablemente cuando ésta presenta pardeamiento enzimático en su estructura; la destrucción de los compartimentos cloroplásticos y las vacuolas provocan la liberación de sus contenidos lo que hace posible la reacción enzimática (Liao et al., 2006; Morante et al., 2014).

Las polifenol oxidasas son enzimas que usan el oxígeno molecular para convertir los fenoles en quinonas (Mayer, 2006; Tran et al., 2012). La oxidación de los sustratos fenólicos es catalizada por las enzimas PPOs que da como resultado la formación de un complejo proteico y la aparición de manchas cafés compuestas por melanina en la estructura de los frutos. Aunque las funciones específicas de las PPOs en los frutos intactos no están esclarecidas; se sugiere que su principal acción en los frutos con cierto tipo de daño es la defensa contra herbívoros y patógenos, incluso con una disminución de su contenido nutricional. Adicionalmente, se ha evidenciado que las PPOs responden a estímulos como el estrés abiótico (Boeckx et al., 2015).

No obstante, la distribución de las PPOs es amplia, existen plantas de varias familias de interés económico que contienen una gran cantidad de PPOs en su constitución y que se presentan a manera de isoenzimas, cuya función se desconoce; entre las cuales se menciona Solanum tuberosum (papa), Glycine max (soya), Solanum melongena (berenjena), Theobroma cacao (cacao). En algunas plantas se ha observado que los niveles de fenoles presentes en su estructura aumentan en el momento de la recolección. Sin embargo, en el momento de la maduración los niveles de PPOs se mantienen casi constantes (Morante et al., 2014).

Theobroma cacao es una planta de interés nacional, puesto que implica una decena de actores en los procesos iniciales como siembra y mantenimiento de la planta hasta la cosecha y elaboración de productos procesados. Las zonas de producción del cacao son las regiones de la Costa y Amazonía en Ecuador, siendo de mayor enfoque la provincia de Los Ríos cuya fuente principal de ingresos proviene del agro (Guerrero, 2014). De acuerdo al Boletín emitido por el Ministerio de Comercio Exterior, se ubica con el 6.69\% el grupo de productos denominado Cacao y elaborados durante el período enero-abril del 2015 en el apartado de exportaciones no petroleras a los principales mercados de Norteamérica,
Europa y Asia; con lo que se deduce su importancia para la economía del país (Ministerio de Comercio Exterior, 2015).

Mediante el uso de aplicaciones biotecnológicas y moleculares se pretende identificar, secuenciar y caracterizar al menos un gen responsable de la síntesis de PPOs en cacao como parte de un estudio pionero en el país que pretende buscar mecanismos biológicos de defensa de las plantas de cacao frente a patógenos.

La identificación de los genes que se encargan de la síntesis de PPOs en cacao supone un aporte significativo para la ciencia y por ende a la economía de Ecuador a través de la resistencia del cacao a las principales enfermedades, especialmente la monilia (causada por el hongo Moniliophthora roreri), que causa maduración prematura, marchitez, secamiento hasta llegar a los abultamientos y la momificación de la mazorca con lo que se presume el contagio del resto de la plantación que puede llegar hasta el $80 \%$ de la plantación (Solis y Suárez, 2006; Villavicencio y Jiménez, 2010).

Para la realización de la investigación se pretende la identificación molecular de al menos un gen codificante de polifenol oxidasa (PPO) en Theobroma cacao L. (cacao) en el cantón Quevedo, provincia de Los Ríos, debido a la importancia del cultivo en la provincia y el país, con el objetivo de mejorar la cadena productiva, los réditos percibidos y la valoración a nivel mundial de este producto.

\section{Materiales y métodos}

\section{Extracción de RNA de hojas de cacao Recolección del material vegetal}

Se usaron hojas de cacao nacional (Theobroma cacao L.) procedentes de árboles seleccionados por sus características sobresalientes a nivel agronómico como productividad y poca incidencia a enfermedades, recolectados en la Finca Experimental La Represa, de la Universidad Técnica Estatal de Quevedo, ubicada en Quevedo, provincia de Los Ríos.

Se trabajó con tejido foliar proveniente de plantas resistentes (clon L8H12) y susceptibles (clon L44h88) de la finca experimental "La Represa", en función de los clones reportados como resistentes y susceptibles a monilia y a otras enfermedades, según Amores et al. (2009) y Sánchez-Mora et al. (2014). Al momento de la recolección, las hojas se sumergieron en nitrógeno líquido y se conservaron a $-80^{\circ} \mathrm{C}$ hasta su uso.

\section{Extracción de DNA genómico de cacao}

Para validar la eficiencia de los oligonucleótidos e identificar posibles intrones en los productos de amplificación, se realizó la extracción y purificación de DNA genómico de cacao en función de DNeasy Plant Mini Kit de Qiagen. 
Extracción y purificación de RNA total de cacao de alta calidad

Eliminación de ribonucleasas

Los materiales, soluciones y reactivos se trataron con dietilpirocarbonato (DEPC), inhibidor de RNAasas, a una concentración de $1 \mathrm{ml} \mathrm{L}^{-1}$ de agua.

Método de extracción de RNA descrito por Morante et al. (2014)

Para la extracción de RNA total se trabajó con el protocolo de Morante et al. (2014) para plantas con alto contenido de polisacáridos y polifenoles. Debido a que los tejidos utilizados por Morante et al. (2014), son muy similares al cacao en cuanto a su recalcitrancia y a su dificultad por aislar RNA de alta calidad, se realizó la extracción de RNA de cacao siguiendo las recomendaciones de los autores.

Dentro de las modificaciones están las variaciones de peso de tejido (100-300 mg y 500-1000 mg), adición de tampón de lavado y tampón de extracción a cada muestra de manera individual.

Las hojas provenientes de plantas sanas y enfermas se maceraron con nitrógeno líquido hasta obtener un polvo fino y se añadió buffer de extracción (300Mm Tris- $\mathrm{HCl}, 25 \mathrm{mM}$ EDTA, $2 \mathrm{M} \mathrm{NaCl}, 2 \%$ CTAB, $2 \%$ PVPP, $0.05 \%$ espermidina y $2 \%$ mercaptoetanol antes de usar) precalentado a $65^{\circ} \mathrm{C}$. La proporción de tejido y buffer de extracción fue de 10 a $12 \mathrm{~mL}$ por cada gramo de tejido. El pellet obtenido después de la extracción se precipitó con $\mathrm{LiCl} 10 \mathrm{M}$.

\section{Tratamiento del RNA con DNAasa}

Para eliminar la presencia del DNA genómico asociado a la extracción de RNA, se empleó la enzima DNase I de Invitrogen, que consiste en la digestión de DNAmonocatenario y bicatenario a oligodeoxi-ribonucleótidos con un fosfato en el extremo 5'. Se añadió la muestra de RNA con buffer de reacción, se añadió $1 \mu \mathrm{L}$ de DNase I y se llevó a volumen de $10 \mu \mathrm{L}$ con agua tratada con DEPC. Tras la adición de los componentes se inactivó la enzima y se incubó a $65^{\circ} \mathrm{C}$.

\section{Síntesis de cDNA (DNA complementario) para ensayos de amplificación por RT-PCR}

Para la síntesis de cDNA se usó el juego de reactivos "Super Script III First-Strand Synthesis for RT-PCR" de Invitrogen, siguiendo las instrucciones del fabricante. Como molde se empleó el RNA total de cacao tratado con DNAse I para el ensayo de retrotranscripción (RT) (Cuadro 1).

A esta mezcla se le añadió la siguiente combinación de reactivos 10x RT Buffer, $25 \mathrm{mM} \mathrm{MgCl2,} 0.1 \mathrm{M}$ DTT, RNase OUT y Superscript III RT seguido de incubación a $50{ }^{\circ} \mathrm{C}$ por 50 minutos, 10 minutos a $25^{\circ} \mathrm{C}$ y 50 minutos a $50{ }^{\circ} \mathrm{C}$. Para terminar los tiempos de incubación se incuba 5 minutos a 85 ${ }^{\circ} \mathrm{C}$ para luego reposar en hielo. Por último, se añade $1 \mu \mathrm{L}$ de
Cuadro 1. Síntesis de cDNA a partir de RNA total

\begin{tabular}{lc}
\hline \multicolumn{1}{c}{ Componente } & Cantidad \\
\hline RNA & $\mathrm{n} \mu \mathrm{L}$ \\
$50 \mu \mathrm{M}$ Oligo (dT) & $1 \mu \mathrm{L}$ \\
$10 \mathrm{mM}$ dNTP mix & $1 \mu \mathrm{L}$ \\
Agua-DEPC & Hasta completar la reacción a 10 \\
& $\mu \mathrm{L}$ \\
\hline
\end{tabular}

RNase H y se incuba a $37{ }^{\circ} \mathrm{C}$ por 20 minutos. Se guarda el cDNA sintetizado a $-20^{\circ} \mathrm{C}$.

\section{Reacción en cadena de la polimerasa RT-PCR}

El cDNA sintetizado previamente se utilizó como molde para los ensayos de amplificación. De manera que, se prepararon las reacciones de PCR y se llevaron a cabo en un equipo termociclador Peltier-based y las condiciones de amplificación se ajustaron a las temperaturas de melting (Tm) de los oligonucleótidos y a las especificaciones de uso para la enzima Taq DNA Polymerase Recombinant de Invitrogen.

La mezcla de PCR contenía $10.5 \mu \mathrm{L}$ de agua Milli-Q, $2 \mu \mathrm{L}$ de buffer PCR 10X, $2 \mu \mathrm{L}$ de $\mathrm{MgCl}_{2} 50 \mathrm{mM}, 2 \mu \mathrm{L}$ de dNTPs $10 \mathrm{mM}, 2 \mu \mathrm{L}$ de cada oligonucleótido $10 \mathrm{mM}, 4 \mu \mathrm{L}$ de molde de cDNA y $0.5 \mu \mathrm{L}$ de Taq-polimerasa.

\section{Oligonucleótidos utilizados}

Se diseñaron tres parejas de oligonucleótidos (TcFw4/Tc-Rev4; Tc-Fw5/Tc-Rev5; Tc-Fw6/Tc-Rev6) a partir de secuencias altamente conservadas de PPOs que fueron probados de manera independiente y combinados entre ellos para obtener mayor probabilidad de hibridación, empleando DNA genómico y cDNA como moldes. Todos los oligonucleótidos empleados en este trabajo fueron sintetizados y suministrados por Sigma-Aldrich (Cuadro 2).

\section{Electroforesis de DNA en geles de agarosa}

La separación y validación de los productos de PCR se realizó mediante electroforesis horizontal en geles de agarosa con tinción de bromuro de etidio. Los geles se prepararon en tampón TAE (tris, acetato de sodio, EDTA) 1X que contiene: $40 \mathrm{mM}$ Tris-base; $1 \mathrm{mM}$ EDTA, $20 \mathrm{mM}$ ácido acético.

Los geles se analizaron con un fotodocumentador (E-Gel Imager, Life Technologies) digital que incorpora un transiluminador ultravioleta UVP y una cámara digital de alta resolución.

Purificación de los fragmentos de DNA de geles de agarosa

Los fragmentos de DNA amplificados y separados mediante electroforesis se cortaron con un bisturí, evitando la contaminación entre las bandas. Seguidamente, se purificaron con el kit "Purelink Quick Gel Extraction" de Invitrogen de acuerdo a las instrucciones del fabricante. 
Cuadro 2. Oligonucleótidos empleados en este trabajo

\begin{tabular}{ccl}
\hline Oligonucleótido & $\begin{array}{c}\text { Temperatura de } \\
\text { melting }^{\circ} \mathbf{C}\end{array}$ & \multicolumn{1}{c}{ Secuencia (5'-3') } \\
\hline Tc-Fwd4 & 58.8 & AAAGAAGACGGAATTGTTCA \\
Tc-Fwd5 & 54.7 & GTATTCATGCGTAAAGTAGATC \\
Tc-Fwd6 & 56.9 & TGGCAGATTTGAACTACTTC \\
Tc-Rev4 & 56.9 & CCTTCAATTGTTACACTGCT \\
Tc-Rev5 & 57.6 & GAGGACTCAAATTTGTTTCC \\
Tc-Rev6 & 57.8 & TCCTTCAATTGTTACACTGC \\
\hline
\end{tabular}

\section{Ligación del DNA para clonación}

El inserto/gen amplificado y purificado, se ligó con el vector pCR 2.1 mediante la enzima DNA ligasa proveniente del bacteriófago T4, incorporada en el kit "The Original TA Cloning Kit" de Invitrogen, siguiendo las instrucciones del fabricante.

\section{Transferencia de DNA a Escherichia coli mediante transformación}

Las moléculas obtenidas en la ligación se procedieron a transformar en células de $E$. coli quimio-competentes, estirpe DH5a.

\section{Transformación de $E$. coli y siembra de colonias}

Para la transformación, se empleó el kit One Shot Transformation Protocol, que incorpora el medio SOC (Super Optimal Colony). Al momento de la transformación se dispusieron las células en hielo. Se colocaron $2 \mu \mathrm{L}$ de la reacción de ligación en los viales que contienen las células competentes, se incubaron en hielo por 30 minutos, luego se las sometió a choque térmico por 30 segundos a $42{ }^{\circ} \mathrm{C}$ y se las transfirió a hielo. Se añadieron $250 \mu \mathrm{L}$ de medio SOC a cada vial y se agitaron a $37^{\circ} \mathrm{C}$ por 1 hora a $225 \mathrm{rpm}$.

Pasado el tiempo de la incubación, se dispusieron hasta $200 \mu \mathrm{L}$ de cada transformación a platos de Petri con medio LB (Luria-Bertani), cuyo contenido consta de Bacto triptona, extracto de levadura, cloruro de sodio y bacto agar en incubación a $37^{\circ} \mathrm{C}$ toda la noche.

Para la posterior identificación mediante ensayos de complementación $\alpha$, se suplementó al medio sólido IPTG $0.2 \mathrm{mM}$ y $40 \mu \mathrm{g} \mathrm{mL}^{-1} \mathrm{X}$-gal, de manera que las células transformadas que incorporaron el inserto fueron de color blanco, mientras que, las que no lograron incorporar el inserto fueron de color azul.

\section{Escrutinio de colonias}

Las colonias blancas (que contienen el inserto) se sometieron a ensayos por PCR para la amplificación del fragmento del gen de interés, empleando los primers Tc-Fw6/ Tc-Rev4. Las colonias seleccionadas y comprobadas por PCR se dispusieron a crecimiento en medio LB a $37{ }^{\circ} \mathrm{C}$ toda la noche.

\section{Purificación de DNA plasmídico}

El DNA plasmídico fue purificado mediante el kit PureLink HQ Mini Plasmid Purification, de acuerdo a las instrucciones del fabricante.

\section{Secuenciación de DNA}

La secuenciación de los fragmentos de interés se llevó a cabo en un secuenciador tipo Li-cor por el método de Sanger. Las secuencias de DNA se obtuvieron mediante hibridación con los oligonucleótidos universales M13 forward y M13 reverse. Se secuenció $1 \mu \mathrm{g}$ de DNA plasmídico en un volumen de $15 \mu \mathrm{L}$ de cada muestra.

\section{Caracterización de un gen codificante de polifenol oxidasa} (PPO)

Se utilizó el programa Bio-Edit (USA) para la edición de secuencias y construcción de alineamientos. Para comparar nuestras secuencias contra secuencias de bases de datos públicas se utilizó el programa BLASTX (Altschul et al., 1990), que compara el porcentaje de similitud con otras secuencias de PPOs y se trabaja con el electroferograma proveniente del equipo secuenciador. El alineamiento de secuencias se realizó en el programa CLUSTALW (Thompson et al., 1997) disponible on-line en la página web del European Bioinformatics Institute (www.ebi.ac.uk).

Para la búsqueda y comparación de secuencias de interés se accedió a la base de datos pública NCBI (National Center for Biotechnology Information) y para el alineamiento y traducción de secuencias de DNA a proteínas se accedió a ExPASy (Expert Protein Analysis System).

Se realizó el ensamblaje de secuencias eliminando aproximadamente cincuenta nucleótidos del extremo 3' en dirección Forward y del extremo 5' en dirección Reverse.

\section{Resultados y discusión}

\section{Extracción de RNA total de cacao}

La extracción de RNA de cacao supone un desafío importante para la investigación debido a la complejidad y recalcitrancia del tejido foliar, por lo que se utilizó el método 
Electroforesis de RNA total en geles de agarosa al 1.5\% (p/v) para hojas de cacao provenientes de plantas resistentes y susceptibles a monilia

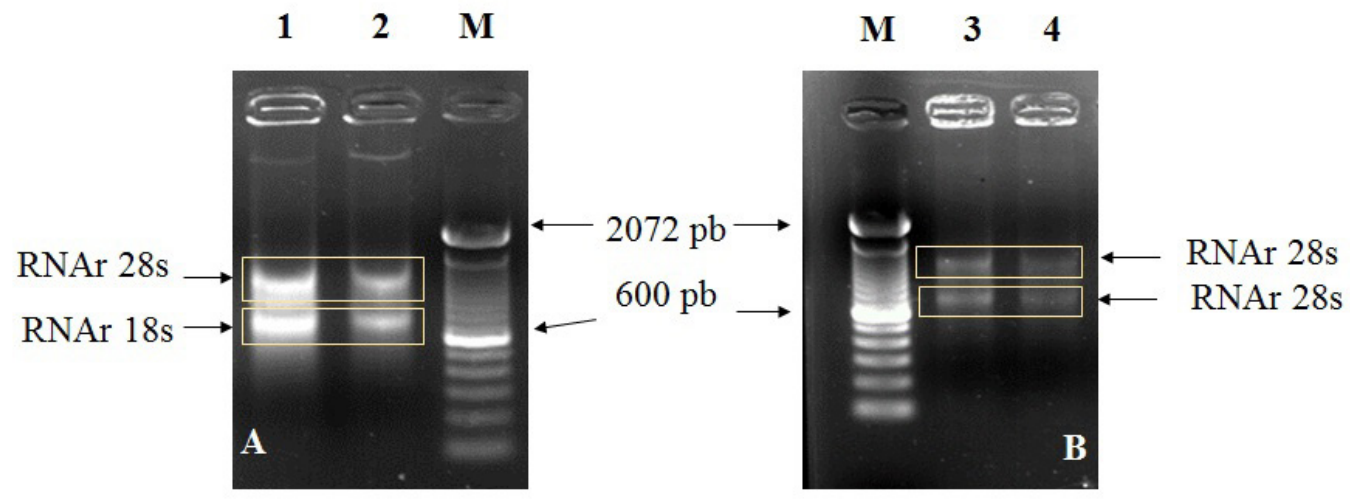

Figura 1. Electroforesis de RNA total en geles de agarosa al $1.5 \%(p / v)$. A, se muestran los productos de extracción de RNA a partir de HPR (calles 1 y 2). B, se muestran los productos de extracción de RNA a partir de HPS a monilia (calles 3 y 4). M, marcador de peso molecular (TrackIt ${ }^{\mathrm{TM}} 100$ pb DNA Ladder, Invitrogen). Se cargó en cada calle $1 \mu \mathrm{L}$ de cada producto de extracción más $2 \mu \mathrm{L}$ de tampón de carga de RNA y $3 \mu \mathrm{L}$ de agua-DEPC

de Morante et al. (2014) para plantas recalcitrantes para conseguir el alto grado de pureza de RNA total imprescindible para la síntesis de cDNA. Este método ha sido eficientemente desarrollado para frutos como el níspero, uvas y rosas con altos niveles de polisacáridos y polifenoles, su aplicación en tejidos de cacao permitió obtener RNA total (Figura 1).

Al momento de la cuantificación por espectrofotometría la proporción A260/A280 evidenció la ausencia de proteínas contaminantes en los productos de extracción, cuyo rango estuvo comprendido entre 1,8-2. La relación 260/230, mostró bajo nivel de contaminación por polisacáridos, carbohidratos y compuestos fenólicos, cuyo rango fue $\geq 2$.

Amplificación de un gen codificante de polifenol oxidasa de cacao

Los ensayos de amplificación se realizaron con oligonucleótidos diseñados a partir del alineamiento múltiple de secuencias predictivas de PPOs en plantas, disponibles en GenBank. De manera específica se favoreció a aquellas secuencias nucleotídicas de PPOs cercanas a la familia botánica del Theobroma cacao L.

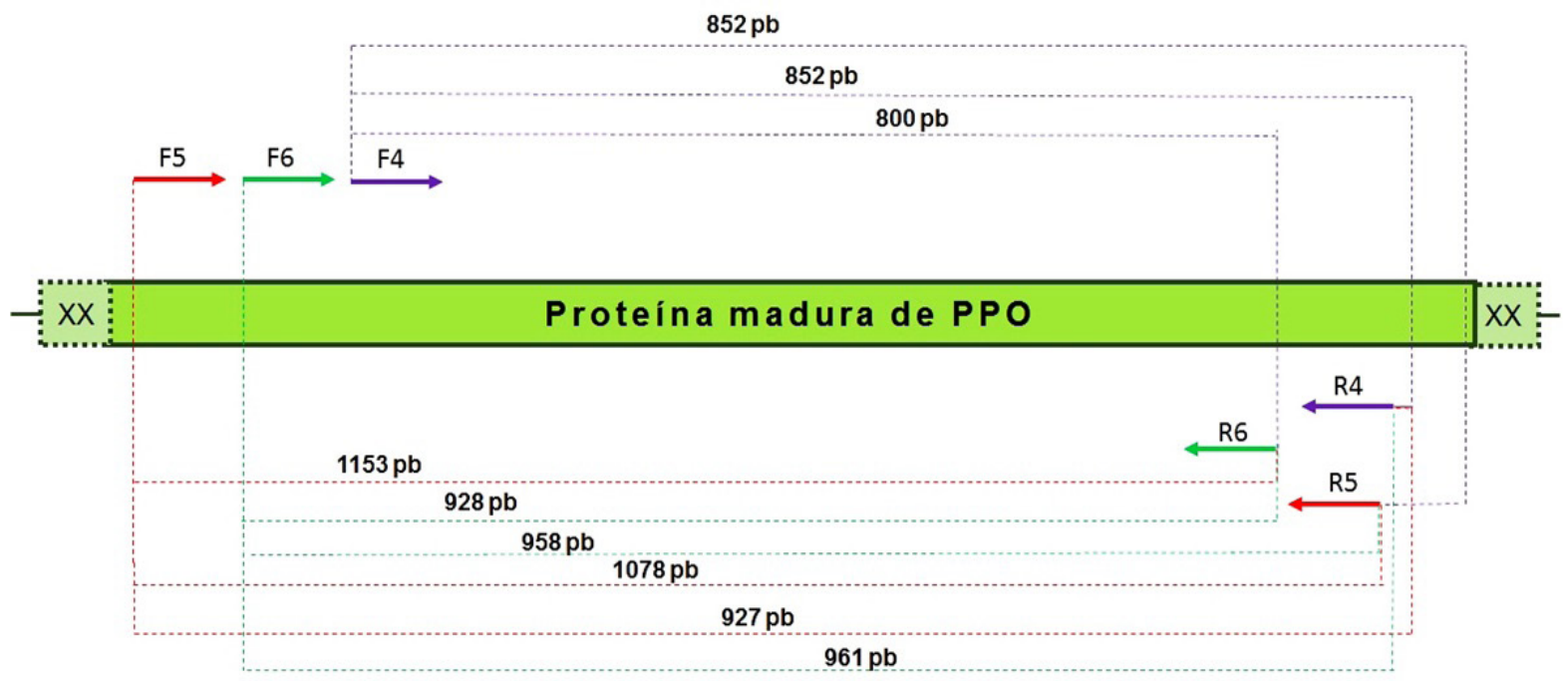

Figura 2. Esquema de orientación de los oligonucleótidos empleados en este trabajo. Las flechas indican la orientación de los oligonucleótidos (Forward y Reverse) y las líneas discontinuas indican la combinación entre ellos. Los números indican el tamaño teórico esperado de los fragmentos 
Las condiciones de amplificación fueron las siguientes (Cuadro 3) una vez que se han definido los oligonucleótidos a usar:

Cuadro 3. Condiciones de amplificación para PCR

\begin{tabular}{lccc}
\hline \multicolumn{1}{c}{ Etapa } & Temperatura & Tiempo & $\mathbf{N}^{0}$ de ciclos \\
\hline $\begin{array}{l}\text { Desnaturalización } \\
\text { inicial }\end{array}$ & $94{ }^{\circ} \mathrm{C}$ & $4 \mathrm{~min}$ & 1 \\
& & & \\
Desnaturalización & $94{ }^{\circ} \mathrm{C}$ & $1 \mathrm{~min}$ & \\
Ligación & $58^{\circ} \mathrm{C}$ & $30 \mathrm{~s}$ & 40 \\
Extensión & $72^{\circ} \mathrm{C}$ & $45 \mathrm{~s}$ & \\
Extensión final & $72^{\circ} \mathrm{C}$ & $7 \mathrm{~min}$ & 1 \\
\hline
\end{tabular}

Se probaron tres parejas de oligonucleótidos (Tc-Fwd4/ TcRev4; Tc-Fwd5/ Tc-Rev5; Tc-Fwd6/ Tc-Rev6) de manera independiente, usando DNAg como molde para comprobar la eficiencia de los oligonucleótidos (Figura 3).

Electroforesis de DNAg en gel de agarosa al 1\% (p/v) para hojas de cacao

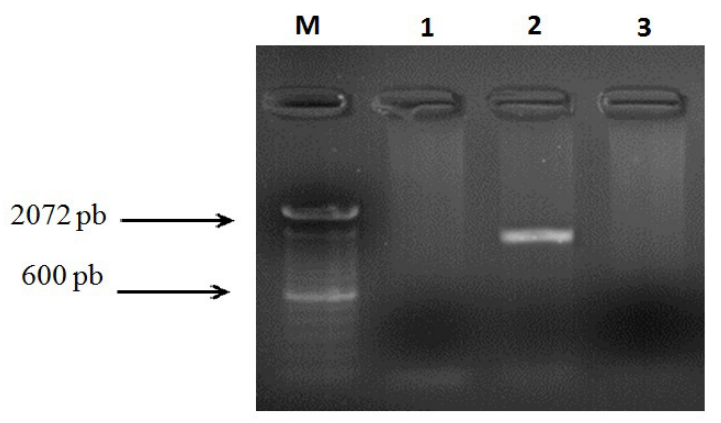

Figura 3. Electroforesis de DNAg en gel de agarosa al $1 \%(p / v)$. Se muestra la amplificación por PCR. Calle 1: Tc-Fwd4 y TcRev4; Calle 2: Tc-Fwd5 y Tc-Rev5; Calle 3: Tc-Fwd6 y Tc-Rev6. M: marcador de peso molecular (TrackIt ${ }^{\mathrm{TM}} 100$ pb DNA Ladder, Invitrogen). Se cargó en cada calle $1 \mu \mathrm{L}$ de cada producto de extracción más $2 \mu \mathrm{L}$ de tampón de carga de DNA y $3 \mu \mathrm{L}$ de agua

El gel de electroforesis usando DNAg como molde muestra la ausencia de productos de amplificación por PCR en las calles 1 y 3 , a diferencia de la calle 2 . Como estrategia para aumentar la capacidad de hibridación, se probaron varias mixturas de oligonucleótidos; Tc-Fwd4/ Tc-Rev5; Tc-Fwd4/ Tc-Rev6; Tc-Fwd5/ Tc-Rev4; Tc-Fwd5/ Tc-Rev6; Tc-Fwd6/ Tc-Rev4; Tc-Fwd6/ Tc-Rev5 (Figura 4).

La banda que aparece en la calle 3, se debe a una hibridación inespecífica de los oligonucleótidos Tc-Fwd5/ Tc-Rev4 con el DNAg. Por su parte, la banda en la calle 5 coincide con el tamaño teórico esperado (961 pb).
Electroforesis de DNAg en gel de agarosa al 1\% (p/v) para hojas de cacao

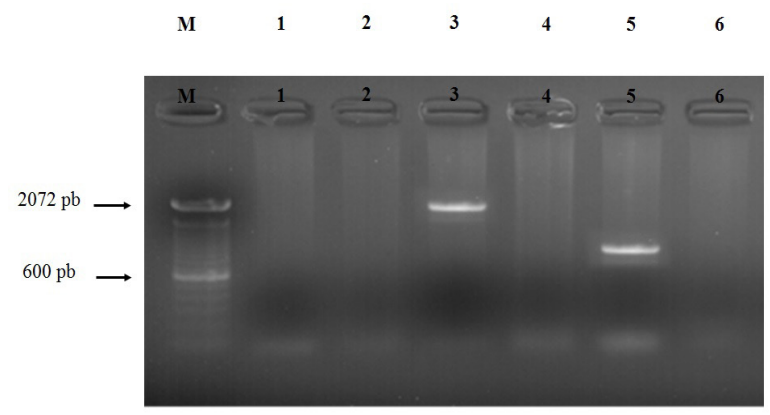

Figura 4. Electroforesis de DNAg en gel de agarosa al $1 \%(\mathrm{p} / \mathrm{v})$. M, marcador de peso molecular (TrackIt ${ }^{\mathrm{TM}} 100$ pb DNA Ladder, Invitrogen); Calle 1: Tc-Fwd4/Tc-Rev5; Calle 2: Tc-Fwd4/TcRev6; Calle 3: Tc-Fwd5/Tc-Rev4; Calle 4: Tc-Fwd5/Tc-Rev6; Calle 5: Tc-Fwd6/Tc-Rev4; Calle 6: Tc-Fwd6/Tc-Rev5. Se cargó en cada calle $1 \mu \mathrm{L}$ de cada producto de extracción más $2 \mu \mathrm{L}$ de tampón de carga de DNA y $3 \mu \mathrm{L}$ de agua.

Únicamente los oligonucleótidos (Tc-Fw5/Tc-Rev5; Tc-Fw6/Tc-Rev4) que hibridaron con el DNA genómico se emplearon en ensayos de amplificación con cDNA, siendo la pareja de oligonucleótidos Tc-Fw6/Tc-Rev4, la que hibridó de manera específica con el cDNA, obteniéndose un fragmento de 1000 pares de bases (Figura 5).

Electroforesis de cDNA en gel de agarosa al 1\% (p/v) para hojas de cacao provenientes de plantas resistentes y susceptibles a monilia

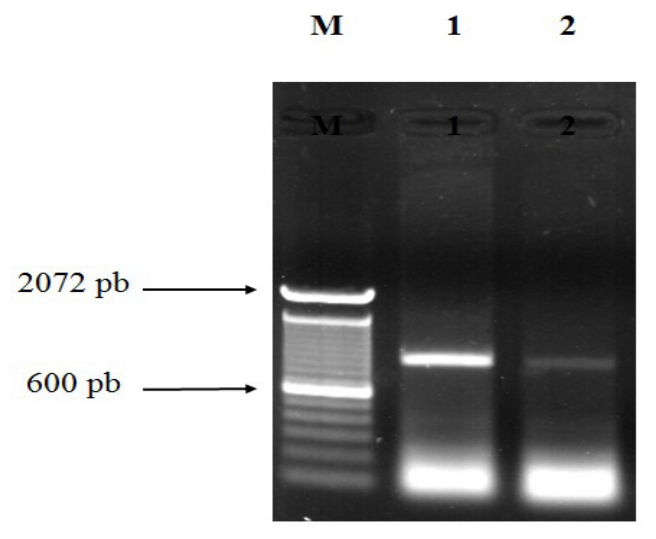

Figura 5. Electroforesis de cDNA con el juego de oligonucleótidos Tc-Fwd6 y Tc-Rev4 en gel de agarosa al 1\% (p/v). M, marcador de peso molecular (TrackIt ${ }^{\mathrm{TM}} 100$ pb DNA Ladder, Invitrogen); Calle 1: cDNA de HPR; Calle 2: cDNA de HPS. Se cargó en cada calle $1 \mu \mathrm{L}$ de cada producto de extracción más $2 \mu$ ILde tampón de carga de DNA y $3 \mu \mathrm{L}$ de agua

Los fragmentos de DNA amplificados y separados por electroforesis, se recortaron en un transiluminador con 
luz ultravioleta y se purificaron con el juego de reactivos "Purelink Quick Gel Extraction" de Invitrogen, de acuerdo a las instrucciones del fabricante. Se comprobaron los productos en geles de agarosa al 1\% (p/v) (Figura 6).

En los carriles 1, 2 y 3 se evidencian bandas de similares pares de bases, provenientes de hoja de planta resistente y susceptible a monilia. La calle 1 muestra la amplificación de DNAg, en contraste con las calles verdes 2 y 3 provenientes de tejido sano y enfermo, respectivamente.

\section{Purificación de DNA plasmídico}

El DNA plasmídico se purificó con el kit PureLink HQ Mini Plasmid Purification Kit. Las secuencias generadas con el vector de clonación sirvieron para la posterior secuenciación, mediante el uso de los primers M13 Forward y M13 Reverse. La eficiencia de la extracción de DNA plasmídico se verificó por amplificación por PCR con el juego de primers Tc-Fw6/ Tc-Rev4 y se visualizó mediante electroforesis horizontal, en el cual aparece una banda de aproximadamente $3000 \mathrm{pb}$, que indica presencia de residuos, y el gen de interés que coincide con el producto amplificado 961 pb (Figura 7).

\section{Secuenciación de DNA}

El método de secuenciación utilizado en este trabajo permitió obtener la secuencia completa del fragmento clonado (Figura 8).

Electroforesis en geles de agarosa 1\% de fragmentos de DNA purificados.

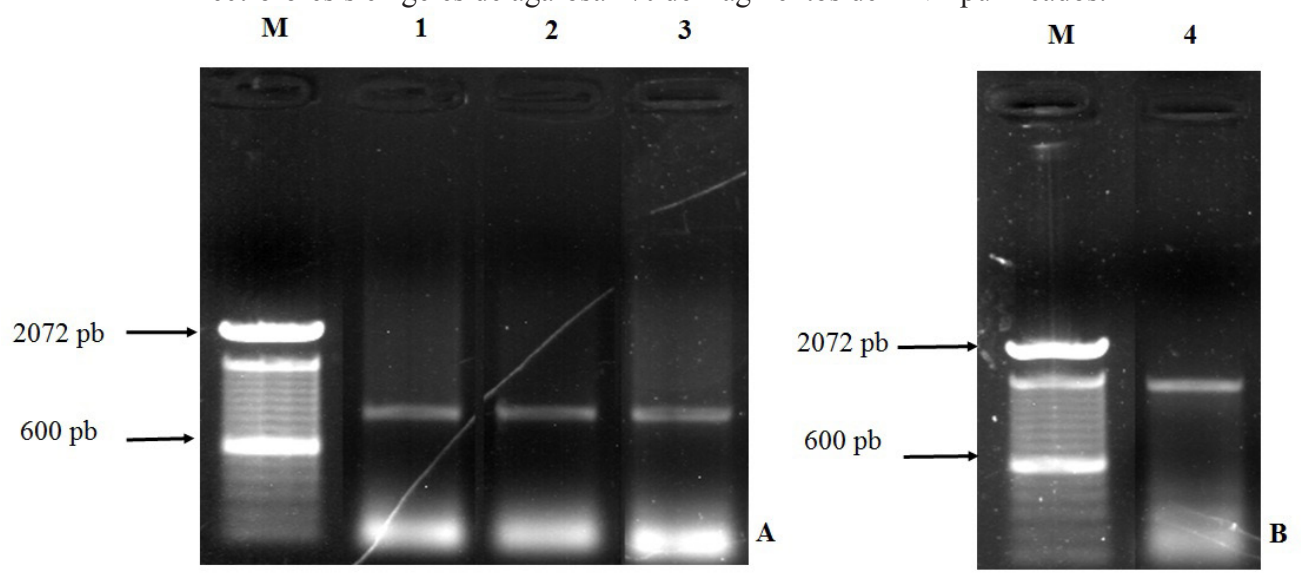

Figura 6. Electroforesis en geles de agarosa $1 \%$ de fragmentos de DNA purificados. A. Juego de oligonucleótidos utilizado Tc-Fwd6 y Tc-Rev4. Calle 1: molde de DNAg, calle 2: molde cDNA de HPR, calle 3: molde de cDNA de HPS. B. Juego de oligonucleótidos usado Tc-Fwd5 y Tc-Rev5. Calle 4: molde DNAg. M: marcador de peso molecular (TrackIt ${ }^{\mathrm{TM}} 100$ pb DNA Ladder, Invitrogen). Se cargó en cada calle $1 \mu \mathrm{L}$ de cada producto de extracción más $2 \mu \mathrm{L}$ de tampón de carga de DNA y $3 \mu \mathrm{L}$ de agua

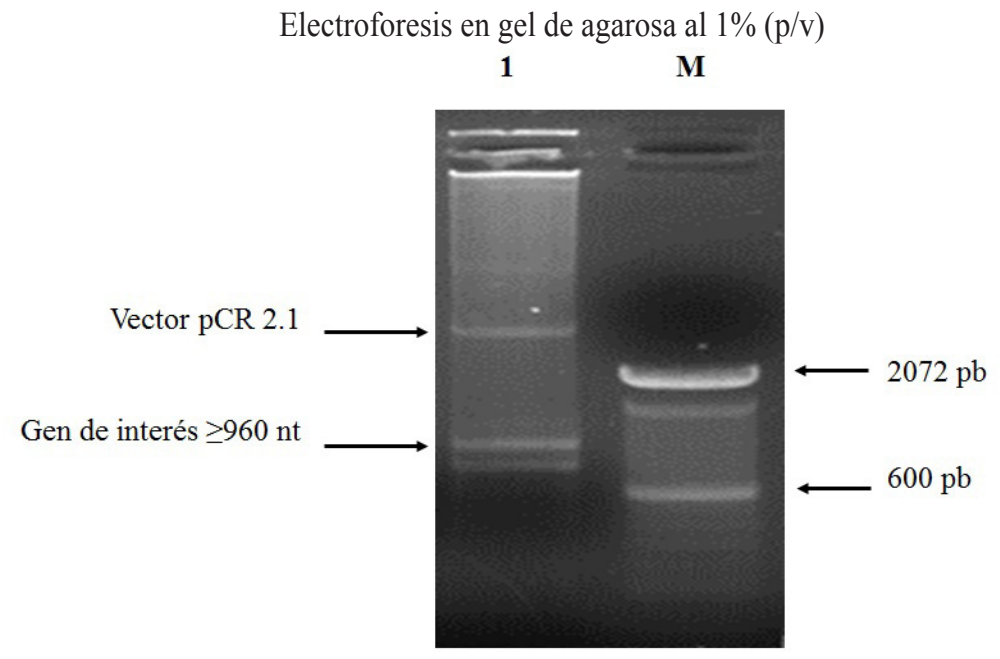

Figura 7. Electroforesis en gel de agarosa al $1 \%(\mathrm{p} / \mathrm{v})$. Calle 1: producto de amplificación de DNA plasmídico purificado. M: marcador de peso molecular (TrackIt ${ }^{\mathrm{TM}} 100$ pb DNA Ladder, Invitrogen) 


\begin{abstract}
Secuencia del gen PPO1-Tc
CTAGCTATGGCTTCTCCTGTCCTGCCGTCATCAACCCCAACCACTATTTCCAGCTCCCCCATTCAAACTTCCTT CTTTCCAAAAACATGTCAACTTTTCTTGAATAAGAAGATCAAAAAGCCAGACTACTCCGGCCCAAGTAAAGT AGTGTCATGCAAAGCCACGAACAATGGGAGCAAAGGAGACTCTTCTCTCAACAGGTTTGATAGAAGGGATC TCCTGATTGGGCTAGGAGGTCTCTACGGGGCAACCATCCTTTCCAATGACCCATTTGCATTAGCAGCACCTAT TGCCGCTCCTGATTTGACCCTTTGCGGTGATGCAACAATATCAGACACAACAAAAGAGACAGTCTATTGCTG CCCACCAGAAACGACAAAGGTCATAGATTTCAAACCACCTACATATTCCAAGATACGCTATAGGTCTGCTGCA CATTTAGTTGATCCTGACTACTTGGCTAAATTTACCAAGGCCATGGAGCTCATGAGAGCTCTTCCTGATGATG ATCCACGTAGTTTCAAGCAACAAGCAAATATTCATTGTGCCTATTGTAACGGGGCTTATGATCAAGTAGGGTT TGATCAAGATATTCAAGTTCATTTCTCGTGGCTCTTTTTCCCATTCCATAGGTTGTATCTTTATTTCTATGAAAG GATTTTGGGCAAGCTGATTGGTGACCCGGATTTCGCCATGCCATTTTGGAACTGGGATGCCCCTGCAGGCATG CCCATACCTGCCATATATGTAAACCCTAACTCTCCACTCTACGATGACAAGCGCAATGTTAACCATCAGGCACC AAAGGTCGCTGATCTTGATTACAATGGCACTGATAAAGATATAACAGACACAGCAATGGTACAAAGTAATCTC AAGGTCATGTACAAGCAGATGGTGTCTGGTTCAAAGACGGCTTCTCTTTTCCATGGAAAGGTGTACCGTGCT GGCGATAAACCCA
\end{abstract}

Figura 8. Secuencia del gen PPO1-Tc identificado en este trabajo. La secuencia consta de $961 \mathrm{nt}$

\section{Caracterización del gen PPO1-Tc}

El gen PPO1-Tc se caracterizó mediante el uso de herramientas bioinformáticas disponibles en NCBI (National Center for Biotechnology Information) y en EBI (European Bioinformatics Institute). La secuencia nucleotídica del gen PPO1-Tc codifica para una proteína de 317 aminoácidos (Figura 9).

Para comprobar la similitud/identidad del gen obtenido en este trabajo se realizó una búsqueda aleatoria mediante Blastn disponible en NCBI, la cual muestra que el gen denominado PPO1-Tc tiene una similitud cercana al 100\% a la secuencia predictiva de PPO de Theobroma cacao depositada en NCBI (XP_017978715.1). Este gen también fue similar a otras PPOs predictivas de cacao y similar en un $70 \%$ a la PPO predictiva de algodón (Cuadro 4). Para comprobar el máximo parentesco molecular con otras PPOs de plantas cercanas a la familia botánica del cacao, se realizó un alineamiento múltiple de secuencias nucleotídicas con las secuencias predictivas de PPOs de cacao depositadas en NCBI, con el fin de comprobar la correspondencia y posición según su ORF (open reading frames, en español marco abierto de lectura), de acuerdo al programa MUSCLE, versión 3.8, disponible en el sitio web del EBI.

Los aminoácidos codificados por el gen PPO1-Tc, ocupan la posición que aparece sombreada y cabe mencionar que la M (metionina) representa el codón de inicio de la proteína (Figura 10).

\begin{abstract}
Aminoácidos codificados por el gen PPO1-Tc
MASPVLPSSTPTTISSSPIQTSFFPKTCQLFLNKKIKKPDYSGPSKVVSCKATNNGSKGDSSLNRFDRRDLLIGL GGLYGATILSNDPFALAAPIAAPDLTLCGDATISDTTKETVYCCPPETTKVIDFKPPTYSKIRYRSAAHLVDPDY LAKFTKAMELMRALPDDDPRSFKQQANIHCAYCNGAYDQVGFDQDIQVHFSWLFFPFHRLYLYFYERILGKL IGDPDFAMPFWNWDAPAGMPIPAIYVNPNSPLYDDKRNVNHQAPKVADLDYNGTDKDITDTAMVQSNLKV MYKQMVSGSKTASLFHGKVYRAGDKP
\end{abstract}

Figura 9. Aminoácidos codificados por el gen PPO1-Tc

Cuadro 4. Máximo parentesco molecular de la proteína PPO1-Tc con otras PPOs provenientes de plantas

\begin{tabular}{|c|c|c|c|}
\hline Id Gen & Similitud & Identidad & Acc. NCBI \\
\hline \multirow{6}{*}{ PPO1-Tc } & PREDICTED: polyphenol oxidase, chloroplastic [Theobroma cacaol & $99 \%$ & $\underline{X P \quad 017978715.1}$ \\
\hline & Larreatricin hydroxylase isoform 1 [Theobroma cacao] & $98 \%$ & EOY28328.1 \\
\hline & Larreatricin hydroxylase isoform 2 [Theobroma cacao] & $98 \%$ & EOY28329.1 \\
\hline & $\underline{\text { Larreatricin hydroxylase [Theobroma cacao] }}$ & $79 \%$ & EOY28325.1 \\
\hline & PREDICTED: polyphenol oxidase I, chloroplastic [Theobroma cacao] & $79 \%$ & $\underline{X P} \quad 017978713.1$ \\
\hline & PREDICTED: polyphenol oxidase, chloroplastic-like [Gossypium raimondii] & $70 \%$ & $\underline{X P} \quad 012451640.1$ \\
\hline
\end{tabular}


Tripletes codificados para aminoácidos

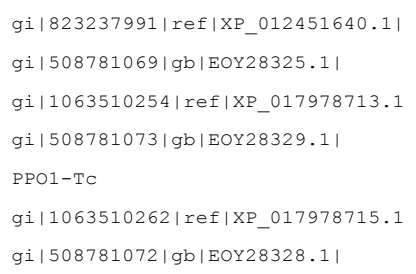

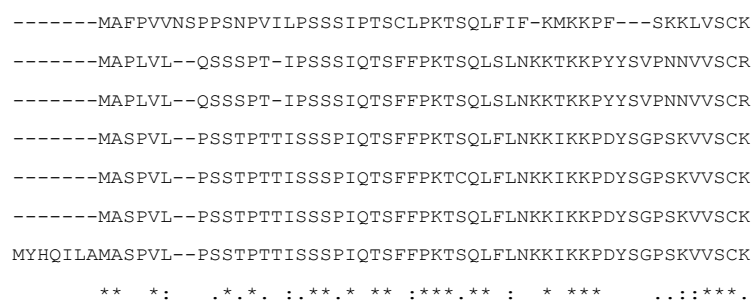

ASNGNQ-------NNEGSSSLNSFDRRDILLGLGSLYGATNLVSDPFALAAPIAAPDLSI ATNNGNQNPTPSSNSKGDSALNRFDRRDLLIGLGGLYGATNLANDPFALAAPIAAPDLTL ATNNGNQNPTPSSNSKGDSALNRFDRRDLLIGLGGLYGATNLANDPFALAAPIAAPDLTI ATNNG-------SKGDSSLNRFDRRDLLIGLGGLYGATNLSNDPFALAAPIAAPDLTL ATNNG-------SKGDSSLNRFDRRDLLIGLGGLYGATILSNDPFALAAPIAAPDLTI ATNNG--------SKGDSSLNRFDRRDLLIGLGGLYGATNLSNDPFALAAPIAAPDLTL ATNNG--------SKGDSSLNRFDRRDLLIGLGGLYGATNLSNDPFALAAPIAAPDLTL

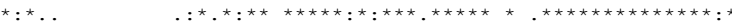

CENSTVTSSSSGTSISVPCCPPKTTNILDFKP PRFSTIRLRPAAHLVDANYLEKFTKAME CGNAVISETTGETTY---CCPPTTTTITDYKPPSFSKLRYRPPAHLVDADYLAKFTKAMN CGNAVISETTGETTY---CCPPTTTTITDYKPPSFSKLRYRPPAHLVDADYLAKFTKAMN

CGDATISDTTKETVY---CCPPETTKVIDFKPPTYSKIRYRSAAHLVDPDYLAKFTKAM CGDATISDTTKETVY---CCPPETTKVIDFKPPTYSKIRYRSAAHLVDPDYLAKFTKAME CGDATISDTTKETVY---CCPPETTKVIDFKPPTYSKIRYRSAAHLVDPDYLAKFTKAME CGDATISDTTKETVY---CCPPETTKVIDFKPPTYSKIRYRSAAHLVDPDYLAKFTKAME

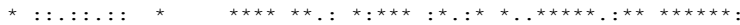

LMKALPDDDPRSFKQQANVHCAYCNGAYDQVGFPDQQLQVHFSWLFF PFHRLYLYFYERI LMKELPSDDPRS FMQQAYVHCAYCNGAYDQVGFPDQDLQVHFSWLFFPFHRWYLYFYERI LMKELPSDDPRSFMQQAYVHCAYCNGAYDQVGFPDQDLQVHFSWLFFPFHRWYLYFYERI LMRALPDDDPRSFKQQANIHCAYCNGAYDQVGF-DQDIQVHFSWLFF PFHRLYLYFYERI LMRALPDDDPRSFKQQANIHCAYCNGAYDQVGF-DQDIQVHFSWLFFPFHRLYLYFYERI LMRALPDDDPRS FKQQANI HCAYCNGAYDQVGF-DQDIQVHFSWLFFPFHRLYLYFYERI LMRALPDDDPRSFKQQANIHCAYCNGAYDQVGF-DQDIQVHFSWLFFPFHRLYLYFYERI

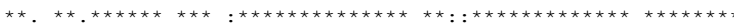

LGKLIGDPNFAMPFWNWDSPPGMAIPKIYVDPNSPLYDEKRNVNHQP PNMLDLDYAGTEE LGKLIGDPDFVMPEWNWDAPAGMPIPAIYVNPQSPLYDDKRNVNHQPPKLADLDYNGTDK LGKLIGDPDFVMPFWNWDAPAGMPIPAIYVNPQSPLYDDKRNVNHQP PKLADLDYNGTDK LGKLIGDPDFAMP FWNWDAPAGMPIPAIYVNPNSPLYDDKRNVNHQPPKVVDLDYNGTDK LGKLIGDPDFAMPFWNWDAPAGMPIPAIYVNPNSPLYDDKRNVNHQAPKVADLDYNGTDK LGKLIGDPDFAMPFWNWDAPAGMPIPAIYVNPNSPLYDDKRNVNHQP PKVADLDYNGTDK LGKLIGDPDFAMPFWNWDAPAGMPIPAIYVNPNSPLYDDKRNVNHQPPKVVDLDYNGTDK

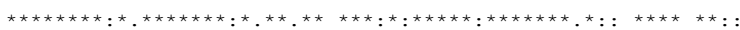

ELSKRDQIKSNLSVMYRQMVT-YKTASLFLGAAYRAGDDPSPGMGSIENNPHTAVHRWVG DITDAEQIAINLKLMYKQMVSNATTATLFHGKPYRAGDAGSPGGGSVELGCHTAIHRWVG DITDAEQIAINLKLMYKQMVSNATTATLFHGKPYRAGDAGSPGGGSVELGCHTAIHRWVG DITDKAMVQSNLKVMYKQMVSGSKTASLFHGKVYRAGDKPSPGGGVVELGSHTAIHRWCG

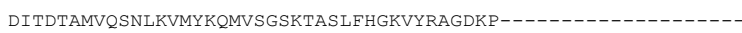
DITDTAMVQSNLKVMYKQMVSGSKTASLFHGKVYRAGDKPSPGGGVVELGSHTAIHRWCG DITDKAMVQSNLKVMYKQMVSGSKTASLFHGKVYRAGDKPSPGGGVVELGSHTAIHRWCG

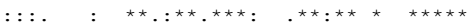

\section{Figura 10. Tripletes codificados para aminoácidos}


Alineamiento múltiple del gen PPO1-Tc

-------MAFPVVNSPPSNPVILPSSSIPTSCLPKTSQLFIF-KMKKPF---SKKLVSCK -------MAPLVL--QSSSPT-IPSSSIQTSFFPKTSQLSLNKKTKKPYYSVPNNVVSCR -------MAPLVL--QSSSPT-IPSSSIQTSFFPKTSQLSLNKKTKKPYYSVPNNVVSCR -------MASPVL--PSSTPTTISSSPIQTSFFPKTSQLFLNKKIKKPDYSGPSKVVSCK ------MASPVL--PSSTPTTISSSPIQTSFFPKTCQLFLNKKIKKPDYSGPSKVVSCK -------MASPVL--PSSTPTTISSSPIQTSFFPKTSQLFLNKKIKKPDYSGPSKVVSCK MYHQILAMASPVL--PSSTPTTISSSPIQTSFFPKTSQLFLNKKIKKPDYSGPSKVVSCK

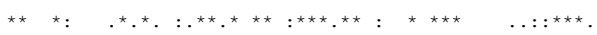

gi|823237991|ref|XP_012451640.1| gi|508781069|gb|EOY28325.1| gi|1063510254|ref|XP_017978713.1 gi|508781073|gb|EOY28329.1| PPO1-TC gi|1063510262|ref|XP_017978715.1 gi|508781072|gb|EOY28328.1|

gi|823237991|ref|XP_012451640.1| gi|508781069|gb|EOY28325.1| gi|1063510254|ref|XP_017978713.1 gi|508781073|gb|EOY28329.1| PPO1-TC

gi|1063510262|ref|XP_017978715.1 gi| 508781072 | gb |EOY28328.1|

gi|823237991|ref|XP 012451640.1 | gi|508781069|gb|EOY28325.1| gi|1063510254|ref|XP_017978713.1 gi|508781073|gb|EOY28329.1| PPO1-TC gi|1063510262|ref|XP_017978715.1 gi| 508781072 | gb|EOY28328.1|

gi|823237991|ref|XP_012451640.1| gi|508781069|gb|EOY28325.1| gi|1063510254|ref|XP_017978713.1 gi|508781073|gb|EOY28329.1| PPO1-TC

gi|1063510262|ref|XP_017978715.1 gi|508781072 | gb|EOY28328.1|

gi|823237991|ref|XP_012451640.1| gi|508781069|gb|EOY28325.1| gi|1063510254|ref|XP_017978713.1 gi|508781073|gb|EOY28329.1| PPO1-TC

gi|1063510262|ref|XP_017978715.1 gi|508781072|gb|EOY28328.1|

Figura 11. Alineamiento múltiple de aminoácidos de otras proteínas con la secuencia traducida del gen PPO1-Tc realizado con el programa Muscle 3.8

ASNGNQ-------NNEGSSSLNSFDRRDILLGLGSLYGATNLVSDPFALAAPIAAPDLSL ATNNGNONPTPSSNSKGDSALNRFDRRDLLIGLGGLYGATNLANDPFALAAPIAAPDLTL ATNNGNQNPTPSSNSKGDSALNRFDRRDLLIGLGGLYGATNLANDPFALAAPIAAPDLTL ATNNG--------SKGDSSLNRFDRRDLLIGLGGLYGATNLSNDPFALAAPIAAPDLTL ATNNG---------SKGDSSLNRFDRRDLLIGLGGLYGATILSNDPFALAAPIAAPDLTL ATNNG---------SKGDSSLNRFDRRDLLIGLGGLYGATNLSNDPFALAAPIAAPDLTL ATNNG---------SKGDSSLNRFDRRDLLIGLGGLYGATNLSNDPFALAAPIAAPDLTL

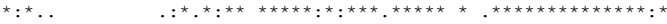

CENSTVTSSSSGTSISVPCCPPKTTNILDFKPPRFSTIRLRPAAHLVDANYLEKFTKAME CGNAVISETTGETTY---CCPPTTTTITDYKPPSFSKLRYRPPAHLVDADYLAKFTKAMN CGNAVISETTGETTY---CCPPTTTTITDYKPPSESKLRYRPPAHLVDADYLAKETKAMN CGDATISDTTKETVY---CCPPETTKVIDFKPPTYSKIRYRSAAHLVDPDYLAKFTKAM CGDATISDTTKETVY---CCPPETTKVIDFKPPTYSKIRYRSAAHLVDPDYLAKFTKAME CGDATISDTTKETVY---CCPPETTKVIDFKPPTYSKIRYRSAAHLVDPDYLAKFTKAME CGDATISDTTKETVY---CCPPETTKVIDFKPPTYSKIRYRSAAHLVDPDYLAKFTKAME

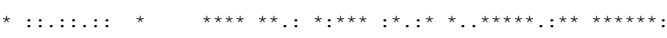

LMKALPDDDPRSFKQQANVHCAYCNGAYDQVGGFPDQQLQVHFSWLFFPFHRLYLYFYERI LMKELPSDDPRSFMQQAYVHCAYCNGAYDQVGFPDQDLQVHFSWLFFPFHRWYLYFYERI LMKELPSDDPRS FMQQAYVHCAYCNGAYDQVGGFPDQDLQVHFSWLFFPFHRWYLYFYERI LMRALPDDDPRS FKQQANIHCAYCNGAYDQVGF-DQDIQVHFSWLFFPFHRLYLYFYERI LMRALPDDDPRSFKQQANIHCAYCNGAYDQVGF-DQDIQVHFSWLFFPFHRLYLYFYERI LMRALPDDDPRSFKQQANIHCAYCNGAYDQVGF-DQDIQVHFSWLFFPFHRLYLYFYERI LMRALPDDDPRSFKQQANIHCAYCNGAYDQVGF-DQDIQVHFSWLFFPFHRLYLYFYERI

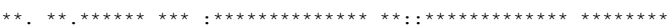

LGKLIGDPNFAMPFWNWDSPPGMAIPKIYVDPNSPLYDEKRNVNHQP PNMLDLDYAGTEE LGKLIGDPDFVMPFWNWDAPAGMP IPAIYVNPQSPLYDDKRNVNHQPPKLADLDYNGTDK LGKLIGDPDFVMPFWNWDAPAGMPIPAIYVNPQSPLYDDKRNVNHQP PKLADLDYNGTDK LGKLIGDPDFAMPFWNWDAPAGMPIPAIYVNPNSPLYDDKRNVNHQPPKVVDLDYNGTDK LGKLIGDPDFAMPFWNWDAPAGMPIPAIYVNPNSPLYDDKRNVNHQAPKVADLDYNGTDK LGKLIGDPDFAMPFWNWDAPAGMPI PAIYVNPNSPLYDDKRNVNHQPPKVADLDYNGTDK LGKLIGDPDFAMPFWNWDAPAGMPIPAIYVNPNSPLIYDDKRNVNHQPPKVVDLDYNGTDK

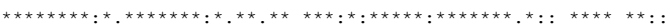

ELSKRDQIKSNLSVMYRQMVT-YKTASLFLGAAYRAGDDPSPGMGS IENNPHTAVHRWVG DITDAEQIAINLKLMYKQMVSNATTATLFHGKPYRAGDAGSPGGGSVELGCHTAIHRWVG DITDAEQIAINLKLMYKQMVSNATTATLFHGKPYRAGDAGSPGGGSVELGCHTAIHRWVG DITDKAMVQSNLKVMYKQMVSGSKTASLFHGKVYRAGDKPSPGGGVVELGSHTAIHRWCG DITDTAMVQSNLKVMYKQMVSGSKTASLFHGKVYRAGDKP--

DITDTAMVQSNLKVMYKQMVSGSKTASLFHGKVYRAGDKPSPGGGVVELGSHTAIHRWCG DITDKAMVQSNLKVMYKQMVSGSKTASLFHGKVYRAGDKPSPGGGVVELGSHTAIHRWCG

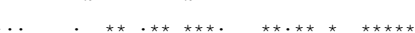




\section{Discusión}

$\mathrm{L}$ a extracción de RNA total de alta calidad en plantas presenta una serie de dificultades, su obtención es determinante para subsecuentes ensayos moleculares como síntesis de DNA complementario (cDNA) mediado por retrotranscriptasa (RT), amplificación por PCR (Birtic y Kranner, 2006), (Islas-Flores et al., 2006), amplificación rápida de DNA complementario final (Portillo et al., 2006) y (Shi y Bressan, 2006); pasos previos e imprescindibles para la clonación, secuenciación y caracterización de un gen codificante de polifenol oxidasa (PPO) de cacao.

Así mismo, la extracción de RNA de tejidos de plantas recalcitrantes conlleva una serie de problemas por la presencia de pectinas, taninos (ME., 1992), pigmentos (Kim y Hamada, 2005), polisacáridos (Manickavelu et al., 2007), poliquinonas (Meisel et al., 2005) y otros metabolitos secundarios (de Keukeleire et al., 2006). Todos estos componentes vegetales pueden interactuar con los ácidos nucleicos, concretamente con el RNA formando complejos insolubles. Esta interferencia química puede causar degradación y bajos rendimientos de RNA mensajeros (RNAm) como un mecanismo de oxidación de polifenoles y coprecipitación con polisacáridos (Wang et al., 2000) y (Morante-Carriel et al., 2014).

Algunos de los protocolos descritos para la extracción de RNA de plantas ricas en polisacáridos y compuestos polifenólicos incluyen el uso de pirrolidona y precipitación con etanol, sin embargo, el uso de este reactivo no siempre se convierte en un beneficio para el investigador (MoranteCarriel et al., 2014). La dificultad de extraer RNA de estos tejidos con los métodos convencionales ya descritos, por lo general siempre requiere incluir modificaciones, optimizando procedimientos específicos para ciertos tejidos vegetales. La aplicación de este protocolo permitió la extracción y purificación de RNA de cacao, con suficiente pureza y calidad, que se empleó para la síntesis de cDNA y clonación y secuenciación de un gen de polifenol oxidasa. Además, se espera que las modificaciones efectuadas funcionen en la extracción de RNA en otras plantas de similares características.

En cuanto a la síntesis de cDNA, es notable mencionar que en los últimos años han aparecido en el mercado juegos de reactivos para la síntesis de cDNA para RT-PCR. El kit "SuperScript First-Strand Synthesis System for RT-PCR" de Invitrogen fue usado para la investigación, cuyas reacciones de cDNA fueron utilizadas para los siguientes ensayos de amplificación de secuencias codificantes de PPOs por PCR.

Por otro lado, a pesar de la descripción de familias multigénicas en varias plantas como el tomate (Newman et al., 1993), papa (Thygesen et al., 2004), plátano (Gooding et al., 2001), álamo (Wang y Constabel, 2004) y algunas Rosáceas (Haruta et al., 1999) y la descripción de expresión simultánea de dos o más genes en un mismo estado de desarrollo (Kim et al., 2001), no se ha descrito hasta el momento en cacao la secuencia de un gen que codifique para polifenol oxidasa ni tampoco la disponibilidad de información en el estadio de la planta en el que se expresa. Sin embargo, en este trabajo y debido al método de secuenciación se identificó la totalidad del fragmento clonado.

Posterior a la identificación del fragmento se completó el alineamiento de secuencias que permitió caracterizar de forma molecular al gen de interés, el cual presentó similitud entre los aminoácidos que ocupan una posición concreta en la secuencia. Sin embargo, las diferencias puntuales pueden deberse a polimorfismos de nucleótido simple (SNPs), ya que estas sustituciones pueden ser el resultado de la sustitución de una sola base en los respectivos codones. Estos cambios son posibles porque el material vegetal usado para secuenciar el genoma de cacao tiene múltiples orígenes (Ecuador, Costa de Marfil, entre otros), siendo diferentes al tejido empleado en el presente trabajo (hoja de cacao Nacional), recolectados de la Finca Experimental la Represa en Quevedo, Ecuador.

El aislamiento de esta secuencia indica que existe base génica y evidencias experimentales de la existencia de al menos un gen codificante de polifenol oxidasa presente en hojas de cacao. A partir de este trabajo, el desafío a futuro, será desarrollar oligonucleótidos específicos para iniciar ensayos de expresión génica mediante PCR cuantitativa en diferentes estados de desarrollo de la mazorca, orientadas a analizar el comportamiento de este gen en situaciones de estrés de la planta de cacao.

\section{Conclusiones}

$\mathrm{L}$ a extracción y purificación de RNA total de alta calidad de hojas de cacao provenientes de plantas identificadas como resistentes y susceptibles a monilia fue exitosa aplicando un protocolos establecidos para plantas recalcitrantes, por tanto, el RNA obtenido se usó para la síntesis de cDNA y los posteriores ensayos de amplificación por PCR; de los cuales se obtuvieron tres fragmentos de PPOs de cacao Nacional, el primer producto derivado de DNAg y dos productos derivados de cDNA, provenientes de plantas resistentes y susceptibles. Finalmente, la caracterización molecular del fragmento de interés, llamado de ahora en adelante gen PPO1-Tc que codifica para polifenol oxidasa (PPOs) en cacao Nacional, Theobroma cacao L. fue exitosa logrando identificar el total de pares de bases y aminoácidos codificados por el gen. Con este resultado exitoso se corrobora la presencia de al menos un gen codificante de PPOs en plantas resistentes y susceptibles a monilia. 


\section{Bibliografía}

Altschul, S., Gish, W., Miller, W., \& Lipman, D. (1990). Basic local alignment search tool (BLAST). Molecular Biology Journal, 215(3), 403-410.

Amores, F., Agama, J., Mite, F., Jiménez, J., Loor, G., \& Quiroz, J. (2009). EET 544 y EET 558. Nuevos clones de cacao nacional para la producción bajo riego en la Peninsula de Santa Elena. Boletín Técnico No 134. Quevedo, Ecuador: INIAP.

Birtic, S., \& Kranner, I. (2006). Isolation of high-quality RNA from polyphenol-, polysaccharide- and lipid-rich seed. Phytochemical Analysis, 17(3), 144-148. doi:10.1002/ pca. 903

Boeckx, T., Winters, A., Webb, J., \& Kingston-Smith, A. (2015). Polyphenol oxidase in leaves; is the any significance to the cloroplastic localization? Journal of Experimental Botany, 66(12), 3571-3579. doi:10.1093/ jxb/erv141

Chun, G. H., Chikako, H., Masayuki, K., Zilian, Z., Tomomi, T., \& Takaya, M. (2002). A simple protocol for RNA isolation from fruit trees containing high levels of polysaccharides and polyphenol compounds. Plant Molecular Biology Reporter, 20(69), 69a-69g. doi:10.1007/BF02801935

Constabel, P., \& Barbehenn, R. (2008). Defensive Roles of Polyphenol Oxidase in Plants. En P. Constabel, Induced Plant Resistance to Herbivory (págs. 253-269). Victoria: Springer.

De Keukeleire, J., Roldán-Ruiz, I., Van Bockstaele, E., Heyerick, A., \& De Keukeleire, D. (2006). Efficient extraction of high-quality total RNA from various hop tissues (Humulus lupulus L.). Preparative Biochemistry and Biotechnology, 36(4), 355-362.

Gooding, P., Bird, C., \& Robinson, S. (2001). Molecular cloning and characterisation of banana fruit polyphenol oxidase. Planta, 213, 748-757. doi:10.1007/s004250100553

Guerrero, G. (2014). Revista Líderes. Recuperado el 2016, de El cacao ecuatoriano su historia empezó antes del siglo XV: http://www.revistalideres.ec/lideres/cacaoecuatoriano-historia-empezo-siglo.html

Haruta, M., Murata, M., Kadokura, H., \& Homma, S. (1999). Immunological and molecular comparison of polyphenol oxidase in Rosaceae fruit trees. Phytochemistry, 50, 1021-1025. doi:10.1016/S0031-9422(98)00633-5

Islas-Flores, I., Peraza-Echeverría, L., Cantó-Canché, B., \& Rodríguez-García, C. (2006). Extraction of high-quality, melanin-free RNA from Mycosphaerella fijiensis for cDNA preparation. Molecular Biotechnology, 34(1), 45 50. doi:10.1385/MB:34:1:45

Kim, J. Y., Seo, Y. S., Kim, J. E., Sung, S.-K., Song, K. J., An, G., \& Kim, W. T. (2001). Two polyphenol oxidases are differentially expressed during vegetative and reproductive development and in response to wounding in the Fuji apple. Plant Science, 161(6), 1145-1152. doi:10.1016/S0168-9452(01)00522-2

Kim, S.-H., \& Hamada, T. (2005). Rapid and Reliable Method of Extracting DNA and RNA from Sweetpotato, Ipomoea batatas (L). Lam. Biotechnology Letters, 27(23), 18411845. doi:10.1007/s10529-005-3891-2

Liao, Z., Chen, R., Chen, M., Yang, Y., Fu, Y., Zhang, Q., \& Lan, X. (2006). Molecular Cloning and Characterization of the Polyphenol Oxidase Gene from Sweetpotato. Molecular Biology, 40(907), 907-913. doi:10.1134/ S0026893306060094

ME., J. (1992). An efficient method for isolation of RNA and DNA from plants containing polyphenolics. Nucleic Acids Research, 20(9), 2381. doi:10.1093/nar/20.9.2381

Manickavelu, A., Kambara, K., Mishina, K., \& Koba, T. (2007). An efficient method of purifying high quality RNA from wheat pistils. Colloids and Surfaces B: Biointerfaces, 54(2), 254-258. doi:10.1016/j.colsurfb.2006.10.024

Mayer, A. (2006). Polyphenol oxidases in plants and fungi: Going places? A review. Phytochemistry, 67(21), 23182331. doi:10.1016/j.phytochem.2006.08.006

Meisel, L., Fonseca, B., Gonzalez, S., Baeza-Yates, R., Cambiazo, V., Campos, R., Silva, H. (2005). A rapid and efficient method for purifying high quality total RNA from peaches (Prunus persica) for functional genomics analyses. Biological Research, 38(1), 83-88.

Ministerio de Comercio Exterior. (2015). Boletín Mensual de Comercio Exterior. Recuperado el 2016, de JunioJulio 2015: http://www.proecuador.gob.ec/wp-content/ uploads/2015/07/JULfinal.pdf

Morante-Carriel, J., Obrebska, A., Bru-Martínez, R., Carranza, M., Pico, R., \& Nieto, E. (2014). Distribución, localización e inhibidores de las polifenol oxidasas en frutos y vegetales usados como alimento. Ciencia y Tecnología, 7(1), 23-31.

Morante-Carriel, J., Sellés-Marchart, S., Martínez-Márquez, A., Martínez-Esteso, MJ., Luque, I., \& Bru-Martínez, R. (2014). RNA isolation from loquat and other recalcitrant woody plants with high quality and yield. Analytic Biochemistry, 452(1), 46-53.

Newman, S., Eannetta, N., Yu, H., Prince, J., de Vicente, M., Anskley, S., \& Steffens, J. (1993). Organisation of the tomato polyphenol oxidase gene family. Plant Molecular Biology, 21(6), 1035-1051.

Portillo, M., Fenoll, C., \& Escobar, C. (2006). Evaluation of different RNA extraction methods for small quantities of plant tissue: Combined effect of reagent type and homogenization procedure on RNA quality-integrity and yield. Physiologia Plantarum, 128(1), 1-7. doi:10.1111/ j.1399-3054.2006.00716.x

Reid, K., Olsson, N., Schlosser, J., Peng, F., \& Lund, S. (2006). An optimized grapevine RNA isolation procedure and statistical determination of reference genes for real-time 
RT-PCR during berry development. BMC Plant Biology, 6(27). doi:10.1186/1471-2229-6-27

Sánchez-Mora, F., Zambrano, J., Vera, J., Ramos, R., Garcés, F., \& Vascónez, G. (2014). Productividad de clones de cacao tipo Nacional en una zona del bosque húmedo tropical de la provincia de Los Ríos, Ecuador. Ciencia y Tecnología, 7(1), 33-41.

Shi, H., \& Bressan, R. (2006). RNA Extraction. Methods in Molecular Biology, 323, 345-348. doi:10.1385/1-59745003-0:345

Solis, K., \& Suárez, C. (2006). Uso de Trichoderma spp para control del complejo Moniliasis-Escoba de Bruja del cacao en Ecuador. 15th Conferencia Internacional de Investigación en Cacao. Quevedo, Ecuador: Instituto Nacional Autónomo de Investigaciones Agropecuarias. Recuperado el 2016

Thompson, J., Gibson, T., Plewniak, F., Jeanmougin, F., \& Higgins, D. (1997). The ClustalX windows interface: flexible strategies for multiple sequence alignment aided by quality analysis tools. Nucleic Acids, 25(24), 48764882.

Thygesen, P., Dry, I., \& Robinson, S. (1995). Polyphenol oxidase in potato. A multigene family that exhibits differential expression patterns. Plant Physiology, 109(2), 525-531. doi:10.1104/pp.109.2.525
Tran, L., Taylor, J., \& Constabel, P. (2012). The polyphenol oxidase gene family in land plants: Lineage-specific duplication and expansion. BMC Genomics, 13(395). doi:10.1186/1471-2164-13-395

Villavicencio, M., \& Jiménez, M. (2010). Caracterización morfológica, fisiológica y patogénica de Moniliophthora roreri aislados de cinco provincias de la Costa Ecuatoriana. CICYT. Recuperado el 2016, de http://www.dspace.espol.edu.ec/xmlui/bitstream/ handle/123456789/10957/Caracterizaci\%C3\%B3n\%20 morfol $\%$ C3\%B3 gica $\% 2 \mathrm{c} \% 20$ fisiol $\% \mathrm{C} 3 \%$ B3 gica $\% 20$ $\mathrm{y} \% 20$ patog $\% \mathrm{C} 3 \% \mathrm{~A} 9$ nica.pdf? sequence $=1 \&$ isAllowed $=\mathrm{y}$

Wang, J., \& Constabel, P. (2004). Polyphenol oxidase overexpression in transgenic Populus enhances resistance to herbivory by forest tent caterpillar (Malacosoma disstria). Planta, 220(1), 87-89. doi:10.1007/s00425004-1327-1

Wang, S., Hunter, W., \& Plant, A. (2000). Isolation and purification of functional total RNA from woody ranches and needles of Sitka and white spruce. BioTechniques, 28(2), 292-296. 